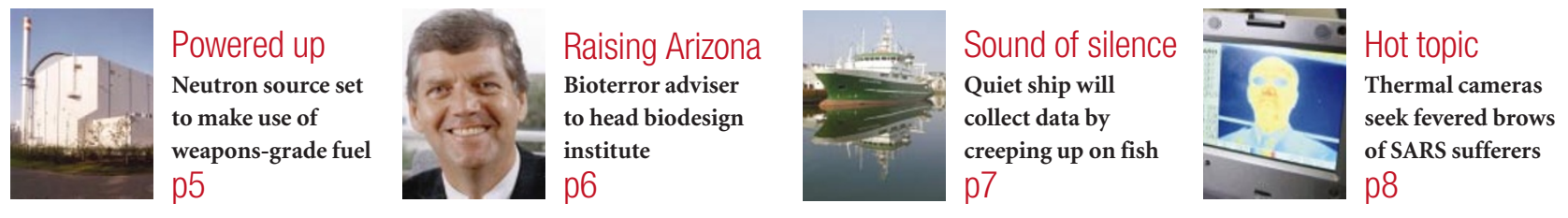

\title{
Apartment complex holds clues to pandemic potential of SARS
}

\section{David Cyranoski, Tokyo} and Alison Abbott, Munich

A 33-floor apartment block in the bustling Kowloon district of Hong Kong could provide clues to the true risk posed by severe acute respiratory syndrome (SARS), epidemiologists say.

Scientists around the world are racing to gather data that will allow epidemiologists to predict whether SARS is likely to become a full-blown pandemic. Their search has led them to investigate exactly what happened when the disease surged through the Hong Kong residential complex in March.

The study, which is being led by Wilina Lim, head of virology at the Hong Kong Department of Health, is the world's only large-scale investigation of the extent to which people without symptoms are carrying SARS - a critical question for those seeking to predict the epidemic's likely reach.

"The key issue is whether symptomless carriers can infect others, and for how long they can do so," says Albert Osterhaus, head of the Netherlands National Influenza Centre in Rotterdam. "If carriers are symptomless for extended periods, a pandemic is quite possible. If not, the epidemic is likely to be controlled."

As of 28 April, 131 cases of SARS had been confirmed among residents of Block $\mathrm{E}$ of the heavily hit Amoy Gardens apartment complex in Kowloon. And 241 symptomless

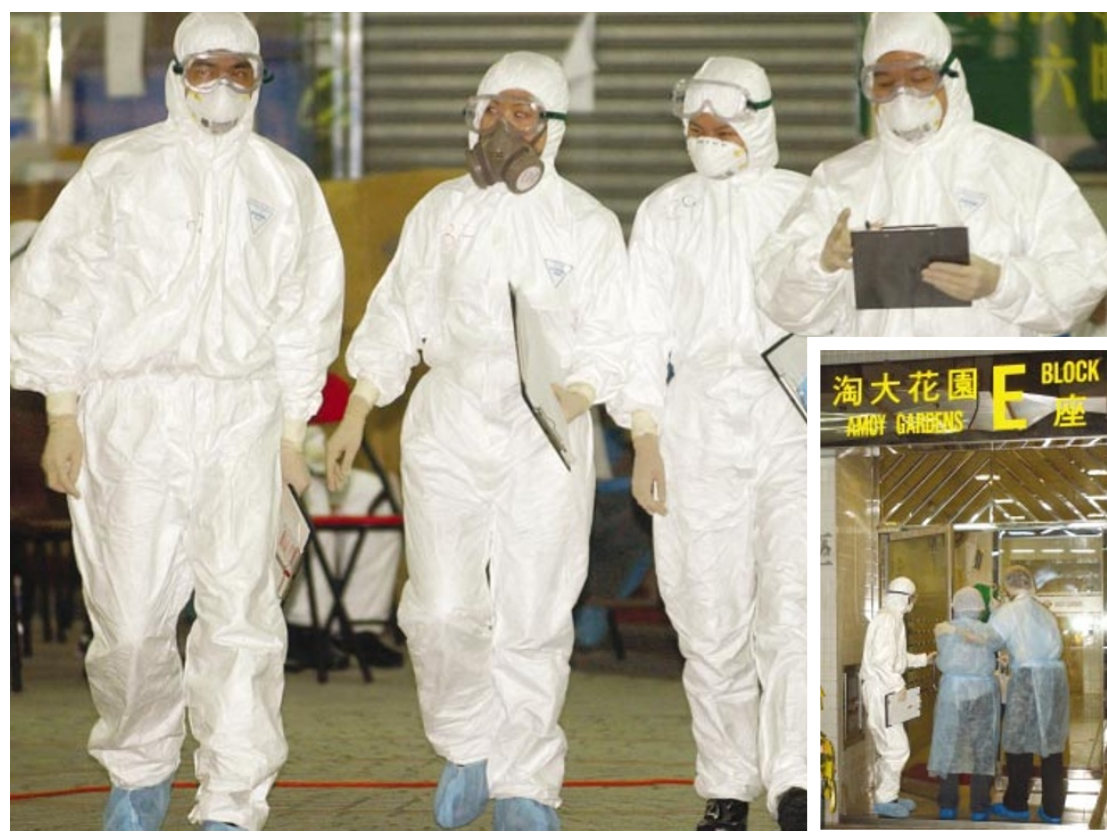

Block $\mathrm{E}$ of the Amoy Gardens residential complex is the focus of a major Hong Kong investigation.

residents were quarantined on 1 April for 10 days following the local outbreak.

Just over 100 of the quarantined residents are taking part in Lim's study, the results of which, she says, should be known by midMay. The researchers are trying to take throat samples from the participants every other day to follow the course of the disease, should it emerge. The samples will be tested for SARS using a version of the polymerase chain reaction (PCR) to amplify and detect genetic material from the coronavirus that is thought to cause the illness. But Lim admits that since the end of the quarantine, sampling has been

\section{Biologists seek to head off future sources of infection}

Researchers at the Beijing Genomics Institute of the Chinese Academy of Sciences have mounted a wide-ranging search of domestic and wild animals, in a hunt for coronaviruses similar to that which is thought to cause severe acute respiratory syndrome (SARS). The work is part of a broader international effort that is attempting to seal off potential reservoirs of future infection.

"Dozens of species have been tested," says the institute's director, Henry Yang, although he declines to reveal which ones, and whether they have been found to be free of coronavirus. "If we said we haven't found anything yet in, say, chickens, people would rule out the chicken as the reservoir," he says. "But that would be jumping the gun."

The closest known relatives of the human SARS virus are coronaviruses from cows and birds, species that Yang says his group is treating as priorities. Coronaviruses have also been found in pigs, mice and cats. But the relationship between these animal viruses and the human SARS virus is relatively distant so Yang also intends to look in other species, such as bats and monkeys, to see whether a closer relative can be found.

Meanwhile, other researchers are looking at the possibility that SARS is transmitted in blood - which would make blood banks potential reservoirs. As yet, they do not know whether the virus is present in the blood of symptomless carriers, although most consider this unlikely by extrapolation to other viral infections. Agencies such as the US Food and Drug Administration and the Frankfurt Blood Bank are looking at screening strategies to identify the virus, should it turn up in blood. Alison Abbott and David Cyranosk 
less consistent as the subjects do not always return to the hospital as requested.

Blood samples were also taken from each of the subjects at the beginning of the quarantine period, and they will be taken again this week. These will be tested for the presence of antibodies against the coronavirus, which will show whether the subjects have been exposed to the virus and have mounted a successful defence. Lim is not prepared to confirm how many of the study's subjects have now developed symptoms of the disease.

Data from these samples, as well as from patients suffering from SARS, will offer clues to some of the key biological questions, says Lim, including when the virus starts to show up in bodily fluids, where in the body it comes from, and whether there are symptomless carriers. "At the moment we don't know enough about the route of transmission of the virus," Lim says. "There is just not enough material yet for investigation."

The study has also analysed hundreds of samples taken from surfaces in the Amoy Gardens apartments, such as toilet seats and door handles, some of which have tested positive for SARS in PCR tests.

In the next few weeks, Lim's group will also begin a study on people from outside Amoy Gardens who have had contact with the disease but have yet to display symptoms.

Other researchers involved in the global network established by the World Health Organization (WHO) in response to the SARS outbreak confirm that it will be several weeks, or even months, before they will

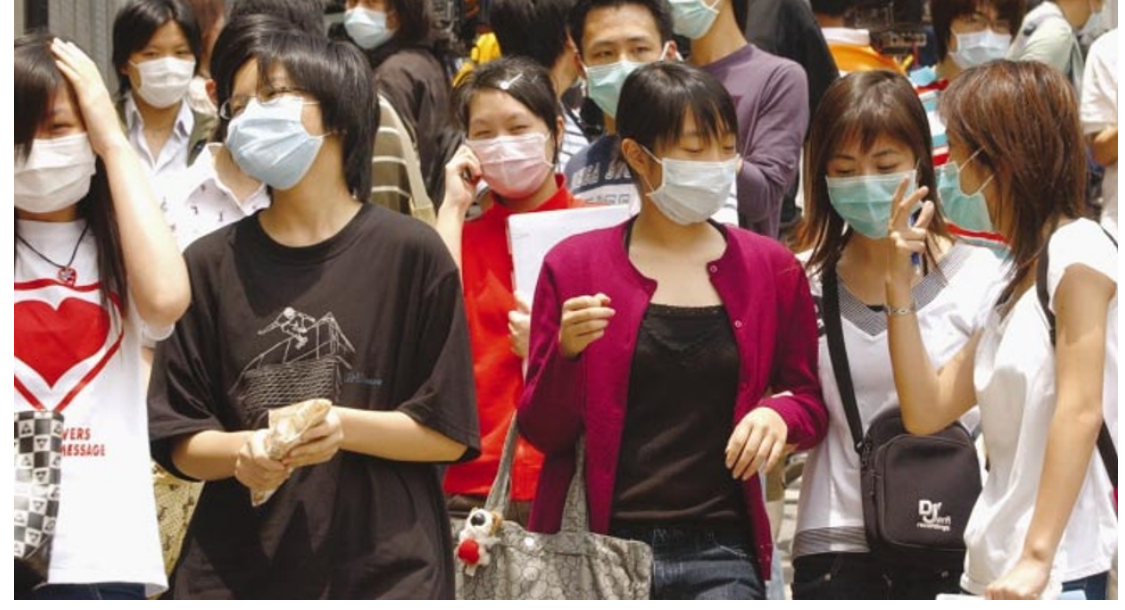

Covered up: students in Kowloon protect themselves following the area's outbreak of SARS.

have enough data to make even the broadest of predictions about the disease's spread.

Samples from patients from countries where SARS infection has been significant, including Hong Kong, Vietnam and Singapore, are also being tested regularly to determine how best to track the virus during the course of the disease. Results of PCR analysis show that stool samples test positive for viral particles for up to 30 days, by which time symptoms have often disappeared. The particles seem to be infective for up to ten days - once isolated they can infect normal cells in culture - but it has not yet been established whether they stay infective after that.

Tracking populations of sick patients and healthy people who have been in contact with them is a powerful method for trying to understand the progress of SARS, but many details can only be investigated in animal models. A monkey model based on macaques, which has just been developed at Erasmus University in Rotterdam, the Netherlands, is now also being used at the US Centers for Disease Control and Prevention in an attempt to understand factors such as how quickly symptoms appear after infection.

But with primates so expensive and awkward to work with, labs in the WHO network are trying to develop models for SARS in more convenient animals such as mice. "We'll need several months to get a suitable small animal model," says Christian Drosten, of the Bernhard Nocht Institute for Tropical Medicine in Hamburg, Germany.

The WHO, meanwhile, has already issued strict travel guidelines, assuming the worst. "We don't have the luxury of seeing what happens and then acting," says David Heymann, the WHO's executive director for communicable diseases.

i www.who.int/csr/sars/travel

\section{Critics slam treatment for SARS as ineffective and perhaps dangerous}

Health authorities in Hong Kong are coming under intense criticism over their use of an antiviral drug combined with anti-inflammatory steroids to treat patients with severe acute respiratory syndrome (SARS).

Critics of the treatment say that there are few indications that the combination of steroids and the antiviral drug ribavirin is effective. And autopsies revealing damage to organs other than the lungs have raised the question of

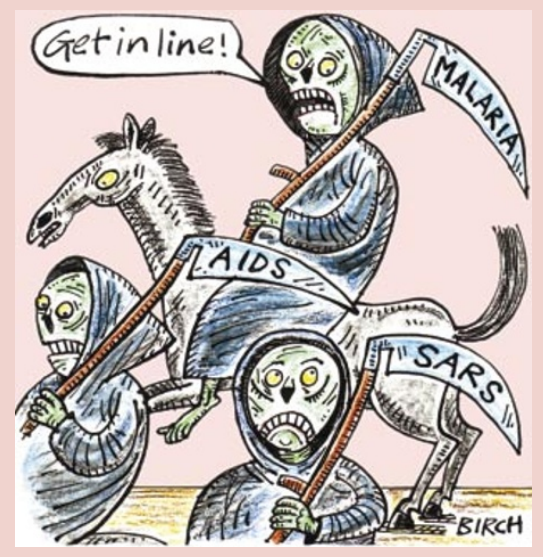

whether the drugs are partly to blame.

"There has been concern in Hong Kong about the efficacy of the treatment, and doctors there are calling for a re-examination of the protocol amid concerns that the prolonged use of highdose ribavirin may be causing or exacerbating multi-organ failure," says Gurinder Shahi, a physician and chairman of the Singapore-based biotechnology company BioEnterprise Asia.

Many in Hong Kong say that the necessary clinical tests have not been done, and that it is therefore too early to judge. "There is no clear evidence either way right now," says Ka-fai To, a pathologist at the Chinese University of Hong Kong whose autopsies of SARS victims found evidence of damage to the lymph nodes and spleen. "These findings could be side effects of the drug, but they could also be the effect of the virus or even an immune response to the virus," she says.

In Hong Kong, which has the largest number of SARS cases and deaths outside mainland China, all patients receive the combination treatment, says John Tam, director of virology at the Prince of Wales Hospital in Hong Kong. The combination is also being used in China's
Guangdong Province, Singapore and Toronto. Although ribavirin is known to be toxic in some patients, it has been prescribed because of its effectiveness against respiratory syncytial virus. This virus is similar to the metapneumovirus that was first isolated in Hong Kong and was originally thought to be the cause of SARS, says Masato Tashiro of the National Institute of Infectious Diseases in Tokyo.

But the drug's efficacy against SARS, which is now thought to be caused by a new strain of coronavirus, is unknown. "Increasing numbers of patients show no improvement," says Tam. In vitro tests are also negative, according to Tashiro.

But in the absence of any obvious alternative, health authorities in Hong Kong are sticking with the therapy. In a speech in Kuala Lumpur on 26 April, the health secretary Carrie Yau Tsang Ka-lai said that $80 \%$ of patients seem to respond favourably to the treatment.

But US officials have rejected ribavirin as a treatment for SARS. At a press briefing on 22 April, Julie Gerberding, director of the Centers for Disease Control and Prevention, said: "There doesn't seem to be any activity of ribavirin against this particular coronavirus." David Cyranoski 